\title{
Throughput and Error State Performance of AMC Scheme in 3G Wireless Systems
}

\author{
KannaRao Shatarasi ${ }^{1}$, SrinivasaRao Reddi ${ }^{2}$, Malothu Amru ${ }^{3}$ \\ Senior Assistant Professor, Aurora's Scientific \& Technological Institute, Hyderabad, AP, India ${ }^{l}$ \\ Associate professor, Aurora's Scientific \& Technological Institute, Hyderabad, AP, India ${ }^{2}$ \\ Associate Professor, Samskruti College of Engineering \& Technology,Hyderabad,AP, India ${ }^{3}$
}

\begin{abstract}
In this paper, Adaptive Modulation and Coding technique for OFDM is proposed. In this technique it is possible to switch the modulation order and coding rate in order to better match the channel conditions. The use of adaptive modulation allows higher throughputs and covering long distances. The aim of this paper is to increase the transmission data rate which the high order modulation scheme with low coding redundancy is used. Numerical results are presented showing that the better performance both in terms of error probability and data throughput.
\end{abstract}

Index terms: OFDMA, adaptive modulation and coding, IEEE 802.16, broadband wireless access, WiMAX.

\section{Introduction}

The use of Adaptive Modulation and Coding (AMC) is one of the key enabling techniques in the standards for 3rd-Generation (3G) wireless systems that have been developed to achieve high spectral efficiency on fading channels [1] - [4]. In the following, we provide a brief description of some of these papers that are more relevant to this current article. Readers are referred to [5] for a more detailed list of references on this topic.

The use of AMC schemes in wireless communication systems is a topic widely considered and investigated in the recent literature [6]-[10]. In [6] the idea of combining the OFDM technique with adaptive modulation and coding is presented by showing the advantages in terms of overall throughput, while in [7], [8] the principles of AMC were proposed and investigated. Successively, in [9], [10] the problem of link adaptation in OFDM system was introduced by considering jointly subcarriers, modulation and power allocation. The MCS is use for the next frame is based on the basic idea of partitioning the estimated channel Signal-to-Noise Ratio (SNR) into regions using a set of threshold values. Each such region is associated with a particular adaptive modulation and coding scheme (MCS) while the threshold values are optimized to maximize the overall throughput. In this paper, we propose a new method for selecting MCS with the objective of maximizing the statistical channel throughput and minimizing the error in predicting the channel SNR.A simplified model with fewer parameters is also proposed, which can be used to account for the changes in the fading characteristics by updating the model parameters in an adaptive manner.

In this paper is organized as follows. The Adaptive modulation and coding technique is presented in Section II. Section III describes the channel state. Section IV explains the error technique. The simulation results are presented in Section V. Concluding remarks are made in Section VI.

\section{Adaptive Modulation And Coding (AMC) Technique}

The block diagram of AMC is shown in Fig.1.

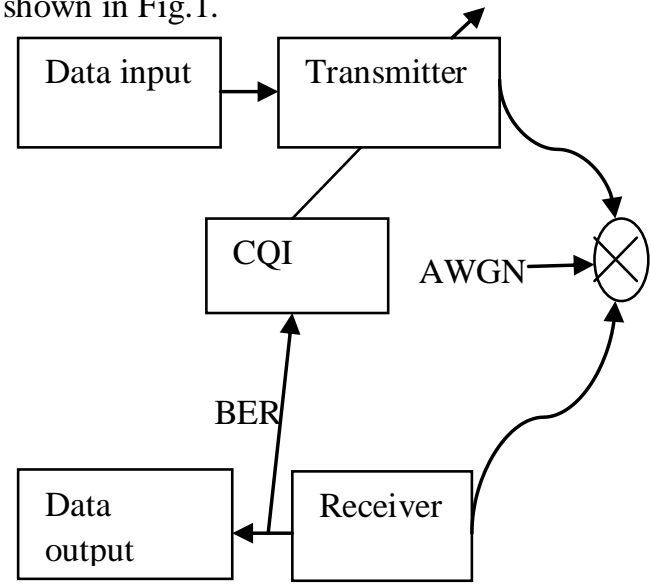

Fig.1. Block diagram of AMC technique 
In AMC technique to changing the modulation and coding scheme to yield a higher throughput by transmitting with high information rates under favorable channel conditions and reducing the information rate in response to channel degradation. In this paper the modulations schemes are QPSK, 16QAM and 64QAM, and codes are of the block type with coding rates $1 / 2$ and 3/4. The AMC technique is follow two approach. The first named channel state and second technique is error state. These techniques are explained in next section.

\section{Channel State}

This technique aims to adapt the modulation and coding scheme after deriving an estimation of the channel behavior in terms of attenuation coefficient. This technique aim is how threshold are derived and their values strongly influence the behavior of the adaptation algorithm in the performance measurement. The channel state technique contains two techniques to derive the threshold as explained below.

\subsection{Maximum throughput (MT) algorithm:}

The MT algorithm aims to maximize the system throughput by selecting for each SNR value the MCS that allows the maximum throughput. The aim is to select the each frame and SNR value, the modulation order and the coding rate that maximize the total throughput. So, it is more efficient scheme in terms of throughput for each $\alpha$ value is selected. Table 1 show that the modulation orders and corresponding $\alpha$ values.

The block probability of MCN can be derived as:

\begin{tabular}{|c|c|}
\hline$\alpha$ value & MCS \\
\hline$\alpha<0.09$ & QPSK 1/2 \\
\hline $0.09<\alpha<0.2$ & QPSK 3/4 \\
\hline $0.2<\alpha<0.33$ & 16QAM 1/2 \\
\hline $0.33<\alpha<0.51$ & 16QAM 3/4 \\
\hline $0.51<\alpha<0.57$ & 64QAM 1/2 \\
\hline $0.57<\alpha$ & 64QAM 3/4 \\
\hline
\end{tabular}

Table 1: MCN selection for the MT approach

$\mathrm{P}_{\text {block }}=1-\mathrm{P}_{\text {cblock }}$

Where

$$
p_{\text {cblock }}=\sum_{i=0}^{t}\left(\begin{array}{l}
N \\
i
\end{array}\right) P_{b}\left(1-p_{b}\right)^{N-i}
$$

The expression of throughput $\eta$ as the function of the channel attenuation $\alpha$ is given by

$$
\eta=\frac{4 .\left(1-\mathrm{P}_{\text {block }} \cdot N_{c} \cdot N_{s d} \log _{2}^{M} \cdot R_{c}\right)}{4 . T}
$$

Where

$\mathrm{M}$ is the modulation order. In this paper $\mathrm{M}=2,4,16$ and $64 . \mathrm{N}_{\mathrm{sd}}$ is the number of OFDM symbols. $\mathrm{T}$ is duration of OFDM frame. $\mathrm{N}_{c}$ is the number of subcarriers per user. $\mathrm{R}_{\mathrm{c}}$ is the adaptive coding rate. $\mathrm{P}_{b}$ is the bit error probability.

\subsection{Target BLER technique:}

The Target BLER (TBLER) algorithm, aims to respect a certain target BLER2 (Block Error Rate) due to specific QoS (i.e., data integrity) requirements. The target value of the BLER can be the same for each SNR or can vary with it. The main feature of the TBLER technique is that of keeping the error rate below a target limit. This technique has one degree of freedom, represented by the imposed target BLER; different values of the target BLER correspond to different system performance. For each value of SNR we can derive BLER as a function of $\alpha$. Consequently, we can define five target $\alpha$ values as shown table.2.

\begin{tabular}{|c|c|}
\hline$\alpha$ value & MCS \\
\hline$\alpha<0.46$ & QPSK 1/2 \\
\hline $0.46<\alpha<0.65$ & QPSK 3/4 \\
\hline $0.65<\alpha<0.95$ & 16QAM 1/2 \\
\hline $0.95<\alpha<1.38$ & 16QAM 3/4 \\
\hline $1.3<\alpha<1.95$ & 64QAM 1/2 \\
\hline $1.95<\alpha$ & 64QAM 3/4 \\
\hline
\end{tabular}

Table 2: MCN selection for the MT approach 
The $\alpha$ values assure the target BLER, for a certain SNR and a certain MCS. Thresholds can be derived by selecting the $\alpha$ value according to the specified Target BLER.

\section{Error Technique}

The error based technique calculates the amount of errors in the downlink subframe. The error technique has been also implemented for different memory depth that corresponds to a different consideration on the erroneous frames. Due to brevity we have focused herein our attention only to the memory depth of four frames. A transition to a more efficient state occurs only if all the last four frames are without errors (when we are the most efficient state (i.e., 64QAM 3/4) we still remain in that state). A transition to less efficient state occurs only if the last frame has at least one error (when we are in the less efficient state (i.e., 4QAM 1/2) we still remain in that state). The state remains the same if the last frame is without errors independently from the previous three.

\section{Experimental Results}

In Fig. 2 it is shown how thresholds (i.e., $\alpha$ values) for state changes are defined in particular herein it is supposed to have a SNR equal to $20 \mathrm{~dB}$. Whenever a different propagation environment is considered it is straightforward to note that, due to different performance in terms of throughput, also the thresholds have different values.

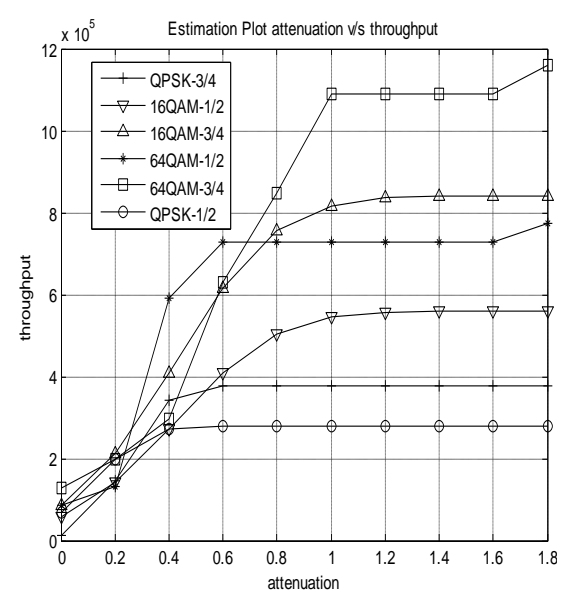

Fig. 2. Thresholds definition in MT technique

Fig. 3 shows BLER behaviors as a function of $\alpha$. This figure highlights how the thresholds are defined under the assumption of a SNR value equal to $16 \mathrm{~dB}$

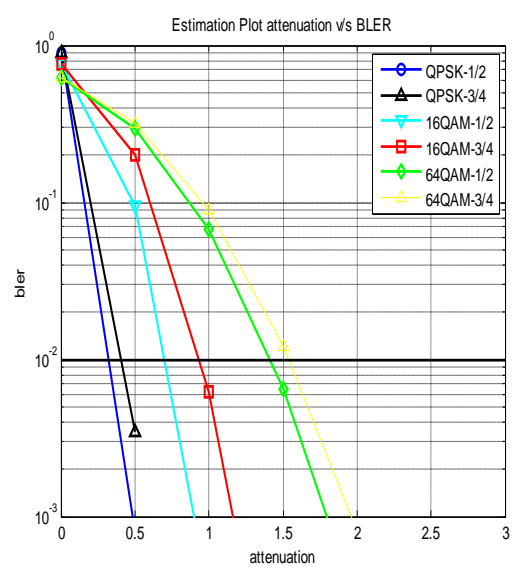

Fig. 3. Thresholds definition in TBLER technique.

Figs. 4 shows performance comparisons in terms of BLER and throughput, respectively, of different AMC techniques based on errors counting with the FMC alternative. 


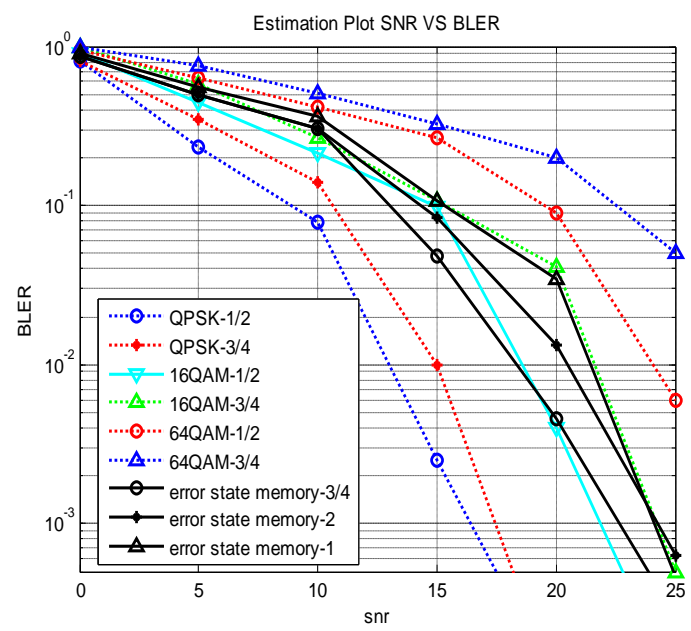

Fig. 4. BLER performance comparison between the error state approach and FMC alternative.

\section{Conclusion}

In this paper, two techniques are used to improve the higher throughput without controlling error rate performance. A frame error rate approach has been then considered by taking into account the effect of the errors in previous frames. The AMC techniques satisfy the different QoS constraints in terms of BLER or throughput. The AMC technique has better performance in error probability and data throughput.

\section{References}

[1] Motorola and Nokia, "Proposal for standardization of very high rate mixed voice-data traffic capabilities, based on extending and enhancing 1X systems," 3GPP2, S00-200003210-020,March, 2000

[2] Motorola and Nokia, "1XTREME," 3GGP2, S00-20000321-019, March, 2000

[3] Motorola, Nokia, Philips, TI and Altera, “Joint 1XTREME Proposal for 1XEV-DV,” 3GPP2, C50-20010611-008a, June, 2001

[4] Motorola, Nokia, Texas Instruments, Altera, and Philips Semiconductors, "1XTREME Physical Specification for Integrated Data and Voice Services in cdma2000 Spread Spectrum Systems," 3GPP2, C50-200010611-013R1, June, 2001.

[5] L. Hanzo, C. H. Wong, M. Yee, Adaptive Wireless Transceivers: Turbo-Coded, Turbo-Equalized and Space-Time Coded TDMA, CDMA, and OFDM Systems. (John Wiley, 2002)

[6] T. Keller and L. Hanzo, "Adaptive modulation techniques for duplex OFDM transmission," IEEE Trans. Veh. Technol., vol. 49, no. 5, pp. 1893-1906, Sept. 2000.

[7] A. J. Goldsmith and S.-G. Chua, "Adaptive coded modulation for fading channels," IEEE Trans. Commun., vol. 46, no. 5, pp. 595602, May1998.

[8] W. T. Webb and R. Steele, "Variable rate QAM for mobile radio," IEEE Trans. Commun., vol. 43, pp. 2223-2230, July 1995.

[9] C. Y. Wong, R. S. Cheng, K. B. Letaief, and R. D. Murch, "Multiuser OFDM with adaptive subcarrier, bit and power allocation," IEEE J. Select. Areas Commun., vol. 17, no. 10, pp. 1747-1758, Oct. 1999.

[10] M.-K. Chang and C.-C. J. Kuo, "Power control, adaptive modulation and subchannel allocation for multiuser downlink OFDM," in Proc. IEEE VTC2004-Fall, Los Angeles, CA, USA, Sept. 2004.

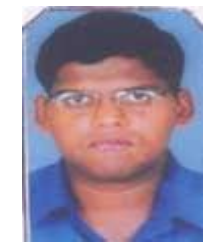

Mr. KANNARAO SHATARASI was born in Warangal, India in 1980. At present he is working as Senior Assistant Professor in Dept of ECE of Aurora's Scientific \& TechnologicalInstitute, Ghatkesar, Hyderabad, Andhra Pradesh, India. He completed B.Tech from JNTUH University. Presently he is doing M.Tech in the area of Communication Systems. His areas of interest are Electro Magnetics, Communication Systems and Electronic Device \& Circuits.

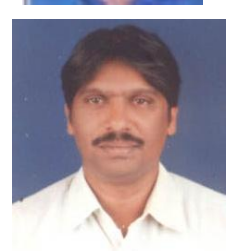

Mr. SRINIVASA RAO REDDI was born in West Godavari, India in 1977. At present he is working as Associate Professor in the Department of ECE, Aurora's Scientific and Technological Institute, Ghatkesar, Hyderabad, Andhra Pradesh, India..He completed Graduation in Engineering, AMIE, The Institution of Engineers (INDIA), Kolkata.M.Tech from JNTUH Hyderabad.His research area includes Signals and Systems, Digital Image Processing and Communication Systems.

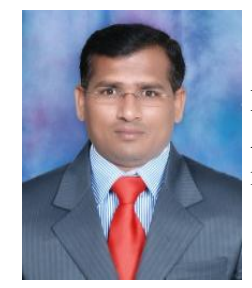

Mr.MALOTHU AMRU was born in Nalgonda,India in 1980. At present he is working as Associate Professor in the Dept of ECE of Samskruti College of Engineering \& Technology, Hyderabad, Andhrapradesh,India He obtained B.Tech from JNTUH University. M.Tech from NIT,Calicut.His areas of interest are Communications and Networks. 